\title{
Multi-Item EOQ Model with Both Demand-Dependent Unit Cost and Varying Leading Time via Geometric Programming
}

\author{
Kotb A. M. Kotb ${ }^{1}$, Hala A. Fergany ${ }^{2}$ \\ ${ }^{1}$ Department of Mathematics and Statistics, Faculty of Science, Taif University, Taif, Saudi Arabia \\ ${ }^{2}$ Department of Mathematical Statistics, Faculty of Science, Tanta University, Tanta, Egypt \\ E-mail: kamkotp2@yahoo.com \\ Received March 10, 2011; revised March 17, 2011; accepted March 21, 2011
}

\begin{abstract}
The objective of this paper is to derive the analytical solution of the EOQ model of multiple items with both demand-dependent unit cost and leading time using geometric programming approach. The varying purchase and leading time crashing costs are considered to be continuous functions of demand rate and leading time, respectively. The researchers deduce the optimal order quantity, the demand rate and the leading time as decision variables then the optimal total cost is obtained.
\end{abstract}

Keywords: Inventory, Geometric Programming, Leading Time, Demand-Dependent, Economic Order Quantity

\section{Introduction}

The problem of the EOQ model with demand-dependent unit cost had been treated by some researchers. Cheng [1] studied an EOQ model with demand-dependent unit cost of single-item. The problem of inventory models involving lead time as a decision variable have been succinctly described by Ben-Daya and Abdul Raouf [2]. AbouEl-Ata and Kotb [3] developed a crisp inventory model under two restrictions. Also, Teng and Yang [4] examined deterministic inventory lot-size models with time-varying demand and cost under generalized holding costs. Other related studies were written by Jung and Klein [5], Das et al. [6] and Mandal et al. [7]. Recently, Kotb and Fergany [8] discussed multi-item EOQ model with varying holding cost: a geometric programming approach.

The aim of this paper is to derive the optimal solution of EOQ inventory model and minimize the total cost function based on the values of demand rate, order quantity and leading time using geometric programming technique. In the final a numerical example is solved to illustrate the model.

\section{Notations and Assumptions}

To construct the model of this problem, we define the following variables:

$D_{r}=$ Annual demand rate (decision variable).

$C_{p r}=$ Unit purchase (production) cost.

$C_{h r}=$ Unit holding (inventory carrying) cost per item per unit time.

$C_{\text {or }}=$ Ordering cost.

$S S=K \sigma \sqrt{L_{r}}=$ Safety stock.

$n=$ Number of different items carried in inventory.

$L_{r}=$ Leading rate time (decision variable).

$Q_{r}=$ Production (order) quantity batch (decision variable).

$T C\left(D_{r}, Q_{r}, L_{r}\right)=$ Average annual total cost. For the $r^{\text {th }}$ item.

The following basic assumptions about the model are made:

1) Demand rate $D_{r}$ is uniform over time.

2) Time horizon is finite.

3) No shortages are allowed.

4) Unit production cost $C_{p r}\left(D_{r}\right)=C_{p r} D_{r}^{-b}$, $r=1,2,3, \cdots, n, b>1$ is inversely related to the demand rate. Where $b$ is called the price elasticity.

5) Lead time crashing cost is related to the lead time by a function of the form $R\left(L_{r}\right)=\alpha L_{r}^{-\beta}, r=1,2,3, \cdots, n$, $\alpha>0, \quad 0<\beta \leq 0.5$. where $\alpha, \beta$ are real constants selected to provide the best fit of the estimated cost func- 
tion.

6) Our objective is to minimize the annual relevant total cost.

\section{Mathematical Formulation}

The annual relevant total cost (sum of production, order, inventory carrying and lead time crashing costs) which, according to the basic assumptions of the EOQ model, is:

$$
\begin{gathered}
T C\left(D_{r}, Q_{r}, L_{r}\right)=\sum_{r=1}^{n}\left[D_{r} C_{p r}\left(D_{r}\right)+\frac{D_{r}}{Q_{r}} C_{o r}\right. \\
\left.+\left(\frac{Q_{r}}{2}+K \sigma \sqrt{L_{r}}\right) C_{h r}+\frac{D_{r}}{Q_{r}} R\left(L_{r}\right)\right]
\end{gathered}
$$

Substituting $C_{p r}\left(D_{r}\right)$ and $R\left(L_{r}\right)$ into (1) yields:

$$
\begin{gathered}
T C\left(D_{r}, Q_{r}, L_{r}\right)=\sum_{r=1}^{n}\left[D_{r}^{1-b} C_{p r}+\frac{D_{r}}{Q_{r}} C_{o r}\right. \\
\left.+\left(\frac{Q_{r}}{2}+K \sigma \sqrt{L_{r}}\right) C_{h r}+\frac{D_{r}}{Q_{r}} \alpha L_{r}^{-\beta}\right]
\end{gathered}
$$

To solve this primal objective function which is a convex programming problem, we can write it in the form:

$$
\begin{aligned}
& \min T C=\sum_{r=1}^{n}\left[C_{p r} D_{r}^{1-b}+\frac{D_{r}}{Q_{r}} C_{o r}\right. \\
& \left.+\left(\frac{Q_{r}}{2}+K \sigma \sqrt{L_{r}}\right) C_{h r}+\frac{D_{r}}{Q_{r}} \alpha L_{r}^{-\beta}\right]
\end{aligned}
$$

Applying Duffin et al. [9] results of geometric programming technique to (3), the enlarged predual function could be written in the form:

$$
\begin{aligned}
G(\underline{W}) & =\prod_{r=1}^{n}\left(\frac{D_{r}^{1-b} C_{p r}}{W_{1 r}}\right)^{W_{1 r}}\left(\frac{D_{r} C_{o r}}{Q_{r} W_{2 r}}\right)^{W_{2 r}}\left(\frac{Q_{r} C_{h r}}{2 W_{3 r}}\right)^{W_{3 r}} \\
& \times\left(\frac{K \sigma C_{h r} \sqrt{L_{r}}}{W_{4 r}}\right)^{W_{4 r}}\left(\frac{D_{r} \alpha L_{r}^{-\beta}}{Q_{r} W_{5 r}}\right)^{W_{5 r}} \\
& =\prod_{r=1}^{n}\left(\frac{C_{p r}}{W_{1 r}}\right)^{W_{1 r}}\left(\frac{C_{o r}}{W_{2 r}}\right)^{W_{2 r}}\left(\frac{C_{h r}}{2 W_{3 r}}\right)^{W_{3 r}} \\
& \times\left(\frac{K \sigma C_{h r}}{W_{4 r}}\right)^{W_{4 r}}\left(\frac{\alpha}{W_{5 r}}\right)^{W_{5 r}} \\
& \times D_{r}^{(1-b) W_{1 r}+W_{2 r}+W_{5 r}} Q_{r}^{-W_{2 r}+W_{3 r}-W_{5 r}} \\
& \times L_{r}^{\frac{1}{2} W_{4 r}-\beta W_{5 r}}
\end{aligned}
$$

Since the dual variable vector $W_{j r}$, $j=1,2,3,4,5, r=1,2,3, \cdots, n$, is arbitrary and can be chosen according to convenience subject to:

$$
W_{1 r}+W_{2 r}+W_{3 r}+W_{4 r}+W_{5 r}=1, W_{j r}>0
$$

We choose $W_{j r}$ such that the exponents of $D_{r}, Q_{r}$ and $L_{r}$ are zero, thus making the right hand side of (4) independent of the decision variables. To do this we require:

$$
\left.\begin{array}{r}
(1-b) W_{1 r}+W_{2 r}+W_{5 r}=0 \\
-W_{2 r}+W_{3 r}-W_{5 r}=0 \\
\frac{1}{2} W_{4 r}-\beta W_{5 r}=0
\end{array}\right\}
$$

These are called the orthogonality conditions which together with (5) are sufficient to determine the values of $W_{j r}, j=1,2,3,4,5, \quad r=1,2,3, \cdots, n$.

Solving (5) and (6) for $W_{j r}$, we get:

$$
\left.\begin{array}{l}
W_{1 r}=\frac{1}{2 b-1}\left\{1-2 \beta W_{5 r}\right\} \\
W_{2 r}=\frac{b-1}{2 b-1}-\left\{1+\frac{2 \beta(b-1)}{2 b-1}\right\} W_{5 r} \\
W_{3 r}=\frac{b-1}{2 b-1}\left\{1-2 \beta W_{5 r}\right\} \quad \text { and } \\
W_{4 r}=2 \beta W_{5 r}
\end{array}\right\}
$$

Substituting $W_{j r}, j=1,2,3,4, r=1,2,3, \cdots, n \quad$ in (4), we get the dual function $g\left(W_{5 r}\right)$. To find $W_{5 r}$ which maximize $g\left(W_{5 r}\right)$, the logarithm of both side of $g\left(W_{5 r}\right)$, and the partial derivatives were taken relative to $W_{5 r}$. Setting it to equal zero and simplifying, we get:

$$
\begin{aligned}
f\left(W_{5 r}\right) & =W_{5 r}^{b_{3}}+A\left(1+2 \beta b_{1}\right) W_{5 r}\left(1-2 \beta W_{5 r}\right)^{b_{2}} \\
& -A b_{1}\left(1-2 \beta W_{5 r}\right)^{b_{2}}=0
\end{aligned}
$$

where:

$$
\begin{aligned}
b_{1} & =\frac{b-1}{2 b-1}, b_{2}=\frac{2 \beta b}{(2 b-1)\left(1+2 \beta b_{1}\right)}, \\
b_{3} & =\frac{1+2 \beta}{1+2 \beta b_{1}} \text { and } \\
A & =\left\{\frac{\alpha}{C_{o r}}\left(\frac{K \sigma C_{h r}}{2 \beta}\right)^{2 \beta}\right\}^{\frac{1}{1+2 \beta b_{1}}} \\
& \times\left[\left\{\frac{2(b-1)}{C_{o r} C_{h r}}\right\}^{b-1} \frac{1}{C_{p r}(2 b-1)^{b}}\right]^{\frac{b_{2}}{b}}
\end{aligned}
$$

It is clear that $f(0)<0$ and $f(1)>0$ which means that there exists a root $W_{5 r} \in(0,1)$. The trial and error method can be used to find this root. However, we shall 
first verify the root $W_{5 r}^{*}$ calculated from (8) to maximize $g\left(W_{5 r}\right)$. This is confirmed by the second derivative to $\ln g\left(W_{5 r}\right)$ with respect to $W_{5 r}$, which is always negative.

Thus, the root $W_{5 r}^{*}$ calculated from (8) maximize the dual function $g\left(W_{5 r}\right)$. Hence, the optimal solution is $W_{j r}^{*}, j=1,2,3,4,5, r=1,2,3, \cdots, n$, where $W_{5 r}^{*}$ is the solution of (8) and $W_{j r^{*}}^{*}, j=1,2,3,4$ are evaluated by substituting value of $W_{5 r}^{*}$ in (7).

To find the optimal values $D_{r}^{*}, Q_{r}^{*}, L_{r}^{*}$, we apply Duffin et al. [9] of geometric programming as indicated below:

$$
\begin{gathered}
C_{p r} D_{r}^{* 1-b}=W_{1 r}^{*} g\left(W_{5 r}^{*}\right), \\
C_{o r} \frac{D_{r}^{*}}{Q_{r}^{*}}=W_{2 r}^{*} g\left(W_{5 r}^{*}\right), \\
C_{h r} \frac{Q_{r}^{*}}{2}=W_{3 r}^{*} g\left(W_{5 r}^{*}\right), \text { and } \\
C_{h r} K \sigma \sqrt{L_{r}^{*}}=W_{4 r}^{*} g\left(W_{5 r}^{*}\right)
\end{gathered}
$$

By solving these relations, the optimal demand rate is given by:

$$
D_{r}^{*}=\left[\frac{C_{o r} C_{h r}}{2 C_{p r}^{2}} \cdot \frac{W_{1 r}^{* 2}}{W_{2 r}^{*} W_{3 r}^{*}}\right]^{\frac{1}{1-2 b}},
$$

The optimal order quantity is:

$$
Q_{r}^{*}=\frac{C_{o r} W_{1 r}^{*}}{C_{p r} W_{2 r}^{*}}\left[\frac{C_{o r} C_{h r}}{2 C_{p r}^{2}} \frac{W_{1 r}^{* 2}}{W_{2 r}^{*} W_{3 r}^{*}}\right]^{\frac{b}{1-2 b}},
$$

The optimal lead time is:

$$
L_{r}^{*}=\left[\frac{C_{o r}}{2 K \sigma C_{p r}} \frac{W_{1 r}^{*} W_{4 r}^{*}}{W_{2 r}^{*} W_{3 r}^{*}}\right]^{2}\left[\frac{C_{o r} C_{h r}}{2 C_{p r}^{2}} \frac{W_{1 r}^{* 2}}{W_{2 r}^{*} W_{3 r}^{*}}\right]^{\frac{2 b}{1-2 b}}
$$

By substituting the values of $D_{r}^{*}, Q_{r}^{*}$ and $L_{r}^{*}$ in (3), we deduce the minimum total cost as:

$$
\begin{aligned}
& \operatorname{minTC}\left(D_{r}^{*}, Q_{r}^{*}, L_{r}^{*}\right)=\sum_{r=1}^{n}\left[C_{p r}\left(\frac{C_{o r} C_{h r}}{2 C_{p r}} \frac{W_{1 r}^{* 2}}{W_{2 r}^{*} W_{3 r}^{*}}\right)^{b_{1}}\right. \\
& \times\left\{1+\frac{W_{2 r}^{*}}{W_{1 r}^{*}}+\frac{\alpha W_{2 r}^{*}}{C_{o r} W_{1 r}^{*}}\left(K \sigma C_{h r} \frac{W_{1 r}^{*}}{W_{2 r}^{*}}\right)^{2 \beta}\right. \\
& \left.\times\left(\frac{2 C_{p r}}{C_{o r} C_{h r}} \frac{W_{2 r}^{*} W_{3 r}^{*}}{W_{1 r}^{*}}\right)^{\frac{2 \beta}{1-2 b}}\right\} \\
& \left.+\frac{W_{1 r}^{*}}{2 W_{2 r}^{*}} \frac{C_{o r} C_{h r}}{C_{p r}}\left(\frac{C_{o r} C_{h r}}{2 C_{p r}^{2}} \frac{W_{1 r}^{* 2}}{W_{2 r}^{*} W_{3 r}^{*}}\right)^{\frac{b}{1-2 b}} \times\left\{1+\frac{W_{4 r}^{*}}{W_{3 r}^{*}}\right\}\right]
\end{aligned}
$$

As a special case, we assume $L_{r}=0, \beta=0$ and $b \rightarrow \infty \Rightarrow R(L)=0, W_{1 r}^{*}=W_{4 r}^{*}=W_{5 r}^{*}=0$, and $W_{2 r}^{*}=W_{3 r}^{*}=\frac{1}{2}$. This is the classical EOQ inventory model.

\section{An Illustrative Example}

We shall compute the decision variables (optimal order quantity $Q_{r}^{*}$, optimal demand rate $D_{r}^{*}$ and optimal lead time $L_{r}^{*}$ ) whose values are to be determined to minimize the annual relevant total cost for three items ( $n$ =3). The parameters of the model are shown in Table 1 .

Assume that the standard deviation $\sigma=6$ unit/year and $K=2$.

For some different values of $\beta$ and $b$, we use equation (8) to determine $W_{5 r}^{*}$, whose value is to be determined to obtain $W_{j r}^{*}, j=1,2,3,4, r=1,2$, 3 from (6).

It follows that the optimal values of the production batch quantity $Q_{r}^{*}$, demand rate $D_{r}^{*}$, lead time $L_{r}^{*}$ and minimum annual total cost are given in Tables 2-7.

Table 1. The parameters of the model.

\begin{tabular}{ccccc}
\hline$r$ & $C_{\text {or }}$ & $C_{p r}$ & $C_{h r}$ & $\alpha_{r}$ \\
\hline 1 & $\$ 200$ & $\$ 10$ & $\$ 0.8$ & $\$ 1$ \\
2 & $\$ 140$ & $\$ 08$ & $\$ 0.5$ & $\$ 2$ \\
3 & $\$ 100$ & $\$ 05$ & $\$ 0.3$ & $\$ 3$ \\
\hline
\end{tabular}

Table 2. The optimal solution of $Q_{r}^{*}$ and $D_{r}^{*}$ as a function of $b$ ( for all $\beta$ ).

\begin{tabular}{ccccccc}
\hline$b$ & $Q_{1}^{*}$ & $Q_{2}^{*}$ & $Q_{3}^{*}$ & $D_{1}^{*}$ & $D_{2}^{*}$ & $D_{3}^{*}$ \\
\hline 2 & 28.21 & 31.17 & 33.10 & 1.450 & 1.550 & 1.540 \\
5 & 26.41 & 28.55 & 31.00 & 1.395 & 1.455 & 1.440 \\
8 & 25.65 & 27.49 & 29.90 & 1.316 & 1.350 & 1.340 \\
10 & 25.25 & 26.99 & 29.40 & 1.280 & 1.300 & 1.295 \\
20 & 24.20 & 25.72 & 28.03 & 1.170 & 1.180 & 1.178 \\
\hline
\end{tabular}

Table 3. The optimal solution of $L_{r}^{*}$ and $\min T C$ as a function of $b(\beta=0.1)$.

\begin{tabular}{ccccc}
\hline$b$ & $L_{1}^{*}$ & $L_{2}^{*}$ & $L_{3}^{*}$ & $\min T C$ \\
\hline 2 & $8.4 \times 10^{-9}$ & $8.9 \times 10^{-8}$ & $1.3 \times 10^{-6}$ & 59.6932 \\
5 & $4.2 \times 10^{-17}$ & $2.4 \times 10^{-14}$ & $4.9 \times 10^{-11}$ & 66.0704 \\
8 & $2.1 \times 10^{-23}$ & $2.4 \times 10^{-17}$ & $1.0 \times 10^{-12}$ & 71.6195 \\
10 & $1.2 \times 10^{-26}$ & $8.1 \times 10^{-20}$ & $2.7 \times 10^{-13}$ & 83.8121 \\
20 & $3.4 \times 10^{-39}$ & $6.9 \times 10^{-26}$ & $1.5 \times 10^{-14}$ & 422.838 \\
\hline
\end{tabular}

Table 4. The optimal solution of $L_{r}^{*}$ and $\min T C$ as a function of $b(\beta=0.2)$.

\begin{tabular}{ccccc}
\hline$b$ & $L_{1}^{*}$ & $L_{2}^{*}$ & $L_{3}^{*}$ & $\min T C$ \\
\hline 2 & $2.7 \times 10^{-9}$ & $5.1 \times 10^{-8}$ & $6.5 \times 10^{-7}$ & 77.539200 \\
5 & $2.5 \times 10^{-17}$ & $2.2 \times 10^{-14}$ & $2.3 \times 10^{-11}$ & 243.99000 \\
8 & $2.2 \times 10^{-24}$ & $4.3 \times 10^{-19}$ & $3.7 \times 10^{-14}$ & 2906.5400 \\
10 & $7.1 \times 10^{-28}$ & $1.4 \times 10^{-21}$ & $1.7 \times 10^{-15}$ & 15175.300 \\
20 & $6.8 \times 10^{-43}$ & $7.7 \times 10^{-31}$ & $9.2 \times 10^{-20}$ & $1.3203 \times 10^{7}$ \\
\hline
\end{tabular}


Table 5. The optimal solution of $L_{r}^{*}$ and $\min T C$ as a function of $b(\beta=0.3)$.

\begin{tabular}{ccccc}
\hline$b$ & $L_{1}^{*}$ & $L_{2}^{*}$ & $L_{3}^{*}$ & $\min T C$ \\
\hline 2 & $2.6 \times 10^{-9}$ & $2.5 \times 10^{-8}$ & $5.5 \times 10^{-7}$ & 112.62400 \\
5 & $2.4 \times 10^{-17}$ & $2.1 \times 10^{-14}$ & $1.2 \times 10^{-11}$ & 6729.1300 \\
8 & $1.7 \times 10^{-24}$ & $8.7 \times 10^{-20}$ & $4.1 \times 10^{-15}$ & 75148.000 \\
10 & $6.4 \times 10^{-29}$ & $5.4 \times 10^{-23}$ & $2.3 \times 10^{-17}$ & $1.495 \times 10^{7}$ \\
20 & $1.2 \times 10^{-46}$ & $2.8 \times 10^{-35}$ & $1.9 \times 10^{-25}$ & $2.858 \times 10^{12}$ \\
\hline
\end{tabular}

Table 6. The optimal solution of $L_{r}^{*}$ and $\min T C$ as a function of $b(\beta=0.4)$.

\begin{tabular}{ccccc}
\hline$b$ & $L_{1}^{*}$ & $L_{2}^{*}$ & $L_{3}^{*}$ & $\min T C$ \\
\hline 2 & $5.9 \times 10^{-10}$ & $1.2 \times 10^{-8}$ & $2.0 \times 10^{-7}$ & 309.50100 \\
5 & $3.9 \times 10^{-17}$ & $1.6 \times 10^{-14}$ & $5.4 \times 10^{-12}$ & 99640.100 \\
8 & $3.4 \times 10^{-25}$ & $3.9 \times 10^{-20}$ & $4.6 \times 10^{-16}$ & $1.317 \times 10^{8}$ \\
10 & $7.9 \times 10^{-29}$ & $2.9 \times 10^{-24}$ & $8.7 \times 10^{-19}$ & $2.050 \times 10^{9}$ \\
20 & $6.8 \times 10^{-51}$ & $5.0 \times 10^{-41}$ & $1.5 \times 10^{-32}$ & $5.629 \times 10^{18}$ \\
\hline
\end{tabular}

Table 7. The optimal solution of $L_{r}^{*}$ and $\min T C$ as a function of $b(\beta=0.5)$.

\begin{tabular}{ccccc}
\hline$b$ & $L_{1}^{*}$ & $L_{2}^{*}$ & $L_{3}^{*}$ & $\min T C$ \\
\hline 2 & $1.2 \times 10^{-10}$ & $3.6 \times 10^{-9}$ & $7.4 \times 10^{-8}$ & 1013.00000 \\
5 & $6.1 \times 10^{-18}$ & $7.2 \times 10^{-15}$ & $8.4 \times 10^{-12}$ & $2.5485 \times 10^{6}$ \\
8 & $5.2 \times 10^{-26}$ & $6.0 \times 10^{-21}$ & $7.1 \times 10^{-17}$ & $2.2880 \times 10^{10}$ \\
10 & $4.9 \times 10^{-30}$ & $5.6 \times 10^{-25}$ & $6.7 \times 10^{-19}$ & $2.31429 \times 10^{12}$ \\
20 & $4.3 \times 10^{-52}$ & $4.8 \times 10^{-44}$ & $5.7 \times 10^{-38}$ & $2.3888 \times 10^{24}$ \\
\hline
\end{tabular}

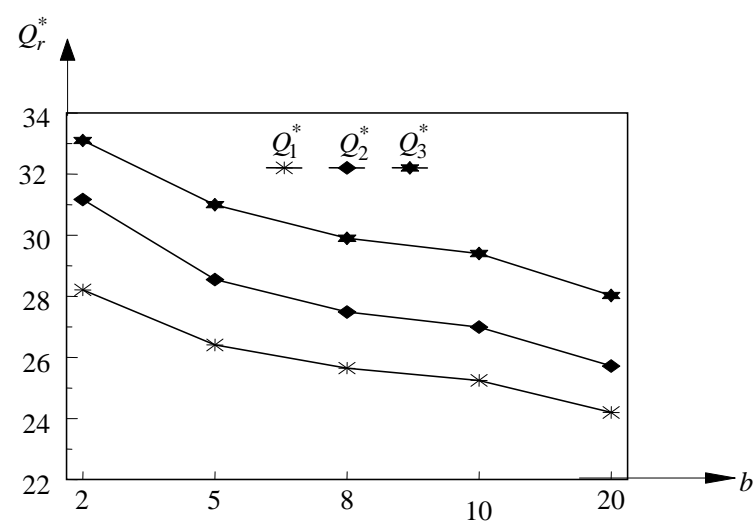

Figure 1. The optimal order quantity against $b$ (for all $\beta$ ).

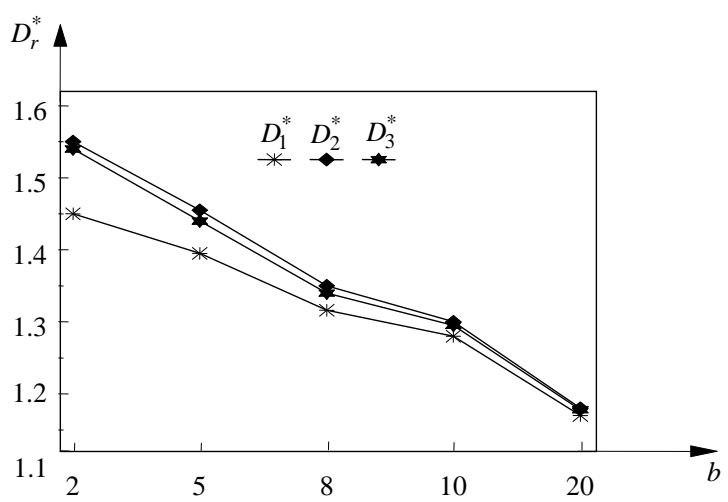

Figure 2. The optimal demand rate against $b$ (for all $\beta$ ). $\min T C \boldsymbol{\Lambda}$

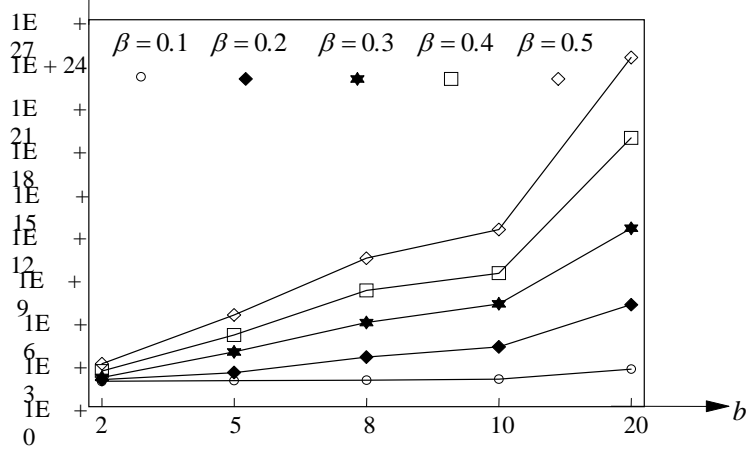

Figure 3. The minimum total cost against $b$ (for all $\beta$ ).

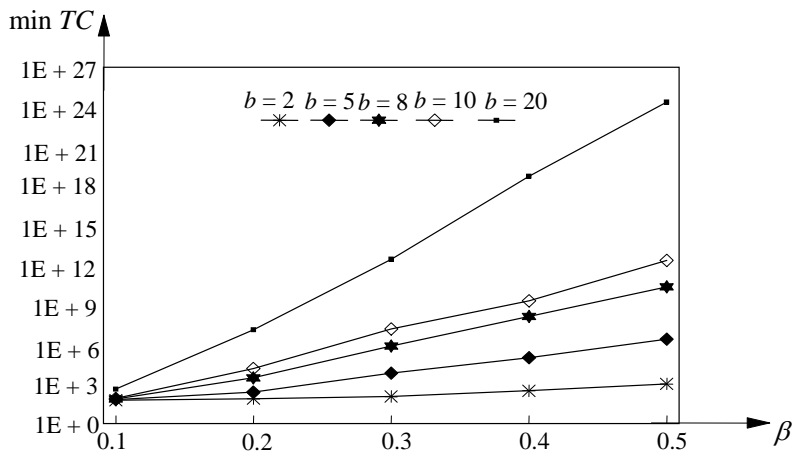

Figure 4. The minimum total cost against $\beta$ (for all $b$ ).

Solution of the problem may be determined more readily by plotting $\left(Q_{r}^{*}, D_{r}^{*}, \min T C\right)$ against $b$ and min TC against $\beta$, for each values of $\beta$.

\section{Conclusions}

This paper is devoted to study multi-item inventory model that consider the order quantity, the demand rate and the leading time as three decision variables. These decision variables $Q_{r}^{*}, D_{r}^{*}$ and $L_{r}^{*}, r=1,2,3, \cdots, n$ are evaluated and the minimum annual total cost min $T C$ is deduced. The classical system is derived as special case and a numerical example is solved.

The smallest value of the minimum total cost is found at the smallest values of $b$ and $\beta$.

\section{References}

[1] T. C. E. Cheng, “An Economic Order Quantity Model with Demand-Dependent Unit Cost,” European Journal of Operational Research, Vol. 40, No. 2, 1989, pp. 252256. doi:10.1016/0377-2217(89)90334-2

[2] M. Ben-Daya and A. Raouf, "Inventory Models Involving Lead Time as a Decision Variable," Journal of the Operational Research Society, Vol. 45, No. 5, 1994, pp. 
579-582.

[3] M. O. Abou-El-Ata and K. A. M. Kotb, “Multi-Item EQO Inventory Model with Varying Holding Cost under Two Restrictions: A Geometric Programming Approach,” Production Planning \& Control, Vol. 8, No. 6, 1997, pp. 608-611. doi:10.1080/095372897234948

[4] J. T. Teng and H. L. Yang, "Deterministic Inventory Lot-Size Models with Time-Varying Demand and Cost under Generalized Holding Costs," Information and Management Sciences, Vol. 18, No. 2, 2007, pp. 113-125.

[5] H. Jung and C. M. Klein, "Optimal Inventory Policies under Decreasing Cost Functions via Geometric Programming,” European Journal of Operational Research, Vol. 132, No. 3, 2001, pp. 628-642. doi:10.1016/S0377-2217(00)00168-5

[6] K. Das, T. K. Roy and M. Maiti, "Multi-Item Inventory with Quantity-Dependent Inventory Costs and Demand-
Dependent Unit Cost under Imprecise Objective and Restrictions: A Geometric Programming Approach," Production Planning \& Control, Vol. 11, No. 8, 2000, pp. 781-788. doi:10.1080/095372800750038382

[7] N. K. Mandal, T. K. Roy and M. Maiti, "Inventory Model of Deteriorated Items with a Constraints: A Geometric Programming Approach,” European Journal of Operational Research, Vol. 173, No. 1, 2006, pp. 199-210. doi:10.1016/j.ejor.2004.12.002

[8] K. A. M. Kotb and H. A. Fergany, "Multi-Item EQO Model with Varying Holding Cost: A Geometric Programming Approach,” International Mathematical Forum, Vol. 6, No. 23, 2011, pp. 1135-1144.

[9] R. J. Duffin, E. L. Peterson and C. Zener, "Geometric Programming-Theory and Application,” John Wiley, New York, 1967. 